Artículo

\title{
Rendimiento y capacidad de expansión del grano de maíz en la raza Palomero Toluqueño
}

\author{
Edgardo Bautista-Ramírez ${ }^{1}$ \\ Amalio Santacruz-Varela ${ }^{2 \S}$ \\ Leobigildo Córdova-Téllez ${ }^{2}$ \\ Abel Muñoz Orozco ${ }^{2 \dagger}$ \\ Higinio López-Sánchez ${ }^{3}$ \\ Gilberto Esquivel-Esquivel ${ }^{4}$
}

${ }^{1}$ Campo Experimental Centro Altos de Jalisco-INIFAP. Carretera Tepatitlán-Lagos de Moreno km 8, Tepatitlán de Morelos, Jalisco. CP. 47600. ${ }^{2}$ Colegio de Postgraduados-Campus Montecillo. Carretera México-Texcoco km 36.5, Montecillo, Texcoco, Estado de México. CP. 56230. ${ }^{3}$ Colegio de PostgraduadosCampus Puebla. Boulevard Forjadores de Puebla núm. 205, Santiago Momoxpan, San Pedro Cholula, Puebla, México. CP. 72760. ${ }^{4}$ Campo Experimental Valle de México-INIFAP. Carretera Los Reyes-Texcoco km 13.5, Coatlinchán, Texcoco, Estado de México. CP. 56250.

${ }^{\S}$ Autor para correspondencia: asvarela@ colpos.mx.

\section{Resumen}

Se ha planteado que la raza de maíz Palomero Toluqueño contribuyó en el origen de otras razas y pese a tener capacidad de reventar no se ha potenciado dicha cualidad. El objetivo del presente estudio fue evaluar el rendimiento y la capacidad de expansión de accesiones de esta raza resguardadas en bancos de germoplasma. Se evaluaron 47 accesiones de Palomero Toluqueño, tres materiales en proceso de mejoramiento genético para reventado y como referencia un material comercial. Los experimentos se establecieron en un diseño de bloques completos al azar con dos repeticiones en el ciclo agrícola primavera-verano 2014 en Montecillo, Estado de México y Santa María Zacatepec, Puebla. Las variables evaluadas fueron: rendimiento de grano, volumen de expansión, porcentaje de granos sin reventar, forma de la roseta, pulverización del pericarpio y volumen de palomitas por hectárea. El promedio general de rendimiento fue de $3.38 \mathrm{t} \mathrm{ha}^{-1} \mathrm{y}$ el volumen de palomitas por hectárea de $8.86 \mathrm{~m}^{3} \mathrm{ha}^{-1}$ mientras que el volumen de expansión por muestra, porcentaje de granos sin reventar y el pericarpio pulverizado tuvieron medias de $2.73 \mathrm{~cm}^{3}$ $\mathrm{g}^{-1}, 67.59 \%$ y 3.2 (escala visual 1-5), respectivamente; en contraste, el testigo comercial, para las mismas variables, mostró valores de $19.33 \mathrm{~cm}^{3} \mathrm{~g}^{-1}, 27.49 \%$ y 1.6. Sólo se presentó la forma de roseta tipo mariposa. Los resultados indican que la raza Palomero Toluqueño tiene una baja capacidad de expansión en relación con el material comercial; sin embargo, su rendimiento de grano superó a la media nacional. Las variables evaluadas demuestran la diversidad que existe dentro de la raza.

Palabra clave: maíz reventador, maíz nativo, palomitas, pericarpio, roseta.

Recibido: julio de 2020

Aceptado: septiembre de 2020 


\section{Introducción}

México es considerado centro de origen y uno de los principales centros de diversidad del maíz (Matsuoka et al., 2002; Kato et al., 2009). Se ha planteado que las razas indígenas antiguas de México se encuentran en la base de la escala evolutiva de maíces cultivados; estas razas son Palomero Toluqueño, Chapalote, Nal-Tel y Arrocillo Amarillo, todas ellas con capacidad de reventado (Wellhausen et al., 1951).

Con respecto a maíces palomeros, además de las razas indígenas antiguas, México cuenta con otras razas como Palomero de Jalisco, Palomero de Chihuahua y Reventador, con amplia diversidad dentro de ellas (Ortega et al., 1991). Palomero Toluqueño se considera, ancestro directo de otras razas como Cónico y Pepitilla, se cultiva en altitudes superiores a $2000 \mathrm{~m}$ su ciclo biológico es corto (Wellhausen et al., 1951), siendo éste un mecanismo de escape a las heladas, sus granos revientan al ser expuestos a temperaturas altas (Mauricio et al., 2004).

Sin embargo, actualmente su consumo principal es en tortillas, por lo que varios productores lo siembran junto con materiales harinosos para mejorar la calidad de la masa (Gámez et al., 2014), mientras que el carácter de reventador ha sido ignorado y su capacidad de expansión es reducida con respecto a los materiales comerciales provenientes de Estados Unidos. Santacruz-Varela (2001) registró volúmenes de $1.62 \mathrm{~cm}^{3} \mathrm{~g}^{-1}$ en muestras de $30 \mathrm{~g}$ de grano, en materiales palomeros nativos de México contra $38.86 \mathrm{~cm}^{3} \mathrm{~g}^{-1}$ en los materiales mejorados de los Estados Unidos de América.

Las principales variables para evaluar la capacidad de expansión en maíces palomeros son: volumen de producto obtenido por unidad de peso de grano, forma de la roseta, persistencia del pericarpio después de la explosión (Ziegler, 2001), así como la proporción de granos sin reventar (Soylu y Tekkanat, 2007), todas ellas influenciadas por factores físicos de la semilla, como grosor y dureza del pericarpio (Hoseney et al., 1983) y humedad del grano (Gökmen, 2004) y por factores químicos como contenido de amilosa y amilopectina (Borras et al., 2006).

A pesar de la importancia evolutiva y de su capacidad de producción en ambientes desfavorables, no existen trabajos dirigidos a potenciar a las razas de maíz palomero de México. Estas razas han sido desatendidas al no haber realizado mejoramiento para reventado del grano, haciendo que su expresión sea baja con respecto a la de sus similares de otros países; por lo anterior, el objetivo de este trabajo fue evaluar el rendimiento y la capacidad de expansión en accesiones de la raza de maíz Palomero Toluqueño, resguardadas en bancos de germoplasma de México y por agricultores custodios de dicha raza, como punto de partida para el diseño de programas de mejoramiento genético de la misma, se plantea como hipótesis que dentro de la diversidad resguardada en bancos de germoplasma y materiales propiedad de los productores custodios existen algunas poblaciones de maíz apropiadas para iniciar un programa de mejoramiento, lo que permitiría en el mediano plazo obtener materiales que compitan con los maíces palomeros comerciales tanto en rendimiento como en su capacidad de expansión. 


\section{Materiales y métodos}

\section{Material genético}

Se evaluaron 51 materiales, 42 correspondientes a accesiones de la raza Palomero Toluqueño de ellas 38 fueron proporcionadas por los bancos de germoplasma y cuatro se obtuvieron de los custodios de la raza Palomero Toluqueño originarios de San Marcos Tlazalpán, Estado de México, además de ocho accesiones misceláneas del programa de mejoramiento genético del Colegio de Postgraduados y un testigo comercial de la marca 'Valle Verde', según se describe en el Cuadro 1.

\section{Cuadro 1. Origen de los materiales evaluados.}

\begin{tabular}{|c|c|c|c|}
\hline \multicolumn{3}{|c|}{ Accesiones } & \multirow{3}{*}{$\begin{array}{c}\text { Donador } \\
\text { Banco de germoplasma } \\
\text { INIFAP }\end{array}$} \\
\hline Mex-5 & Mex-994 & $\begin{array}{l}\text { Pue-956 } \\
\end{array}$ & \\
\hline Mex-6 & Pue-235 & Pue-957 & \\
\hline Tlax-311 & Pue-947 & Pue-958 & \\
\hline Hgo-609 & Pue-948 & Pue-959 & \\
\hline Mex-167 & Pue-949 & Ver-744 & \\
\hline Mex-169 & Pue-950 & Ver-746 & \\
\hline Mex-35 & Pue-951 & Ver-747 & \\
\hline Mex-79\$ & Pue-952 & Ver-748 & \\
\hline Mex-79-Rojo $\$$ & Pue-953 & Ver-749 & \\
\hline Mex-94 & Pue-954 & Ver-750 & \\
\hline Mex-993 & Pue-955 & Ver-751 & \\
\hline ARR-1,2PL (blanco) ${ }^{\&}$ & ARR-76 & ARR-81 & BANGEV-UACh \\
\hline ARR-1,2PL \& & ARR-80 & & \\
\hline $\begin{array}{l}\text { Criollo Encarnación } \\
\text { Criollo Fortino }\end{array}$ & $\begin{array}{c}\text { Criollo Odilón } \\
\text { Criollo Plaza }\end{array}$ & & $\begin{array}{l}\text { Agricultores Custodios, } \\
\text { SINAREFI }\end{array}$ \\
\hline Comp. Amarillo & Palomero Crema & Palomero Tardío & Programa de Mejoramiento \\
\hline Comp. Blanco & Palomero Esbelto & $\begin{array}{l}\text { Palomero Pinto } \\
\text { Amarillo }\end{array}$ & Genético CP \\
\hline NayPP-II × Mex-5-77 & Palomero Grueso & & \\
\hline Valle Verde (testigo) & & & Comercial \\
\hline
\end{tabular}




\section{Ubicación de los experimentos}

Los experimentos se establecieron en campo en el ciclo agrícola primavera-verano de 2014 en Montecillo, municipio de Texcoco, Estado de México (19²7'54.8' latitud norte y $98^{\circ} 54^{\prime}$ '20.5" longitud oeste, altitud de $2250 \mathrm{~m}$, temperatura media anual de $15.1{ }^{\circ} \mathrm{C}$ y precipitación anual de $656.9 \mathrm{~mm}$ ) y en Santa María Zacatepec, municipio de Juan C. Bonilla, Puebla (19 $7^{\circ}$ ' 52" latitud norte y $98^{\circ} 21^{\prime} 23^{\prime}$ " longitud oeste, altitud de $2260 \mathrm{~m}$, temperatura media anual de $16{ }^{\circ} \mathrm{C}$ y precipitación anual $834.9 \mathrm{~mm})(\mathrm{SMN}, 2015)$.

\section{Diseño y unidad experimental}

Se utilizó un diseño de bloques completos al azar con dos repeticiones en cada localidad, la unidad experimental consistió de tres surcos de $5 \mathrm{~m}$ de longitud con surcos de $0.8 \mathrm{~m}$ de ancho, donde se depositaron tres semillas cada $0.5 \mathrm{~m}$ y se aclareó a dos plantas, lo que produjo una población final de 66 plantas por unidad experimental, que corresponde a una densidad de 55000 plantas ha $^{-1}$.

\section{Manejo de los experimentos}

Las siembras se realizaron el 15 y 21 de mayo del 2014 en Montecillo y Santa María Zacatepec, respectivamente. En ambas localidades el cultivo se condujo bajo riego. La fertilización en Montecillo se realizó con la fórmula 140N-60P-00K, aplicando $60 \%$ del N y todo el P en la siembra y el resto del $\mathrm{N}$ en la segunda escarda, mientras que en Santa María Zacatepec la fórmula fue 160N$60 \mathrm{P}-00 \mathrm{~K}$, aplicando $30 \%$ de $\mathrm{N}$ y todo el $\mathrm{P}$ en la primera escarda y el resto del $\mathrm{N}$ en la segunda escarda. La cosecha se efectuó en noviembre de 2014.

\section{Variables respuesta}

Se utilizaron dos de los tres surcos de cada unidad experimental para la evaluación de rendimiento de grano, en el surco restante se realizaron cruzas mesofraternales (Márquez-Sánchez, 2001) para obtener semilla pura y realizar la evaluación de las variables de reventado. Rendimiento de grano (REND). Se determinó en una muestra de cinco mazorcar de cada unidad experimental, con las respectivas correcciones por coeficiente de desgrane y contenido de humedad al $14 \%$. El resultado se reportó en toneladas por hectárea $\left(\mathrm{t} \mathrm{ha}^{-1}\right)$.

Las características de reventado se registraron en granos con contenido de humedad de 13.5\%, por considerarse como óptima para obtener el volumen máximo de expansión (Gökmen, 2004). Para alcanzar esta humedad se colocaron muestras de grano de cada unidad experimental en una cámara de ambiente controlado a $21{ }^{\circ} \mathrm{C}$ con $70 \%$ de humedad relativa durante siete días.

El reventado se realizó en 30 g de semilla colocadas en contenedores de plástico con tapa en hornos de microondas de la marca Daewoo Modelo KOR-164H (127 V y1600 W) programados al 70\% de potencia durante 2:45 min. Se registraron las siguientes variables: volumen de expansión (VE). Se determinó en una probeta circular graduada de $8.89 \mathrm{~cm}$ de diámetro con capacidad de $2000 \mathrm{~mL}$ (Santacruz-Varela, 2001). El resultado se reportó en $\mathrm{cm}^{3} \mathrm{~g}^{-1}$. Porcentaje de granos sin reventar (PGSR). Se dividió el peso de los granos sin reventar entre el peso de la muestra $(30 \mathrm{~g})$ y el resultado se multiplicó por 100. 
Forma de la roseta (FR). Se utilizó una escala visual de 1 a 5, donde 1 corresponde al tipo 'mariposa' (rosetas con protuberancias muy acentuadas de forma irregular) y 5 al tipo 'hongo' (rosetas redondas). Pulverización del pericarpio (PP). Se utilizó una escala visual de 1 a 5, donde 1 corresponde a pericarpio pulverizado en su totalidad y 5 a pericarpio solamente con rupturas leves después de la explosión del grano.

\section{Volumen de expansión por hectárea (VEH)}

Se obtuvo multiplicando el rendimiento por hectárea por el volumen de expansión. El resultado se reportó en $\mathrm{m}^{3} \mathrm{ha}^{-1}$. Se proponen las últimas cuatro variables como alternativas para describir la capacidad de expansión de las accesiones de la raza de maíz Palomero Toluqueño.

\section{Análisis estadístico}

Se realizó análisis de varianza utilizando el modelo lineal del diseño de bloques completos al azar combinando las dos localidades mediante el procedimiento Anova de SAS Versión 9.1 (SAS, Institute, 2004). La comparación de medias se realizó con la prueba de Tukey $(p \leq 0.05)$ y se calcularon los coeficientes de correlación entre variables mediante el procedimiento CORR de SAS Versión 9.1 (SAS Institute, 2004).

\section{Resultados y discusión}

\section{Análisis de varianza}

Las accesiones mostraron diferencias altamente significativas para todas las variables, excepto para pulverización del pericarpio; mientras que las localidades sólo mostraron diferencias significativas para rendimiento y pulverización del pericarpio (Cuadro 2), esto puede ser atribuible a que el rendimiento es un carácter poligénico (Ma et al., 2007) y su comportamiento es altamente influenciado por el ambiente, mientras que el reventado es controlado por una menor cantidad de genes (Lu et al., 2003), haciendo que ésta característica y sus componentes tengan menor influencia ambiental.

Cuadro 2. Cuadrados medios y significancia en el análisis de varianza.

\begin{tabular}{|c|c|c|c|c|c|c|}
\hline \multirow{2}{*}{ Variables } & \multicolumn{3}{|c|}{ Fuentes de variación } & \multirow{2}{*}{$\mathrm{CV}$} & \multirow{2}{*}{$\mathrm{R}^{2}$} & \multirow{2}{*}{ Error } \\
\hline & Localidades (L) & Accesiones (A) & $\mathrm{A} \times \mathrm{L}$ & & & \\
\hline REND & $6.48^{*}$ & $3.38^{* *}$ & $0.76 \mathrm{~ns}$ & 21.76 & 0.81 & 0.54 \\
\hline VEH & $0.56 \mathrm{~ns}$ & $102.11^{* *}$ & $24.51^{* *}$ & 34.84 & 3.09 & 9.54 \\
\hline VE & $1.96 \mathrm{~ns}$ & $31.41^{* *}$ & $1.86^{* *}$ & 24.68 & 0.97 & 0.57 \\
\hline FR & $0.44 \mathrm{~ns}$ & $0.16 \mathrm{~ns}$ & $0.16 \mathrm{~ns}$ & 39.52 & 0.49 & 0.17 \\
\hline PP & $5.83^{* *}$ & $3.22^{* *}$ & $0.03^{*}$ & 16.95 & 0.86 & 0.3 \\
\hline PGSR & $7 \mathrm{~ns}$ & $359.19^{* *}$ & $108.45 \mathrm{~ns}$ & 13.75 & 0.73 & 88.42 \\
\hline
\end{tabular}

**,${ }^{*}=$ significativos a los niveles de 0.05 y 0.01 de probabilidad respectivamente; $\mathrm{ns}=$ no significativo. 


\section{Efecto de los ambientes}

Montecillo, Estado de México fue la localidad con las condiciones que permitieron pulverizar mayor cantidad de pericarpio al momento de reventado, pero a su vez produjo rendimientos inferiores a los obtenidos en Santa María Zacatepec, Puebla (Cuadro 3). Las condiciones de las localidades pudieron haber modificado las concentraciones de celulosa, arabinoxilanos, proteínas estructurales, polímeros fenólicos y lignina, las cuales están relacionados con las características del pericarpio (Bidlack et al., 1992), dichas alteraciones pudieran ser las causantes de la variación en la pulverización del pericarpio de una localidad a otra. Mientras que la diferencia en rendimiento de grano es atribuible a las condiciones climáticas y agroecológicas de las localidades para el mismo año (Buenrostro-Robles et al., 2017).

Cuadro 3. Comparación de medias de dos localidades para las variables evaluadas.

\begin{tabular}{ccccccc}
\hline Localidad & $\begin{array}{c}\text { REND } \\
\left(\mathrm{t} \mathrm{ha}^{-1}\right)\end{array}$ & $\begin{array}{c}\text { VEH } \\
\left(\mathrm{m}^{3} \mathrm{ha}^{-1}\right)\end{array}$ & $\begin{array}{c}\mathrm{VE} \\
\left(\mathrm{cm}^{3} \mathrm{~g}^{-1}\right)\end{array}$ & $\begin{array}{c}\text { FR } \\
(1-5)\end{array}$ & $\begin{array}{c}\text { PP } \\
(1-5)\end{array}$ & $\begin{array}{c}\text { PGSR } \\
(\%)\end{array}$ \\
\hline Sta. María Zacatepec & $3.56 \mathrm{a}$ & $8.916 \mathrm{a}$ & $3.16 \mathrm{a}$ & $1.1 \mathrm{a}$ & $3.41 \mathrm{a}$ & $68.57 \mathrm{a}$ \\
Montecillo & $3.25 \mathrm{~b}$ & $8.8104 \mathrm{a}$ & $2.96 \mathrm{a}$ & 1 & $3.07 \mathrm{~b}$ & $68.2 \mathrm{a}$ \\
DSH $_{0.05}$ & 0.206 & 0.87 & 0.21 & 0.11 & 0.15 & 2.61 \\
\hline
\end{tabular}

Medias con letras iguales en columnas no son estadísticamente diferentes (Tukey $\leq 0.05$ ). DSH= diferencia significativa honesta (0.05).

\section{Efecto de los genotipos}

\section{Rendimiento de grano}

El rendimiento de grano osciló entre 1.05 y $5.14 \mathrm{t} \mathrm{ha}^{-1}$, con un promedio de $3.38 \mathrm{t} \mathrm{ha} \mathrm{h}^{-1}$. Este intervalo, generó nueve grupos estadísticamente diferentes, siendo los valores cercanos al promedio donde se concentró el mayor número de accesiones (Figura 1).

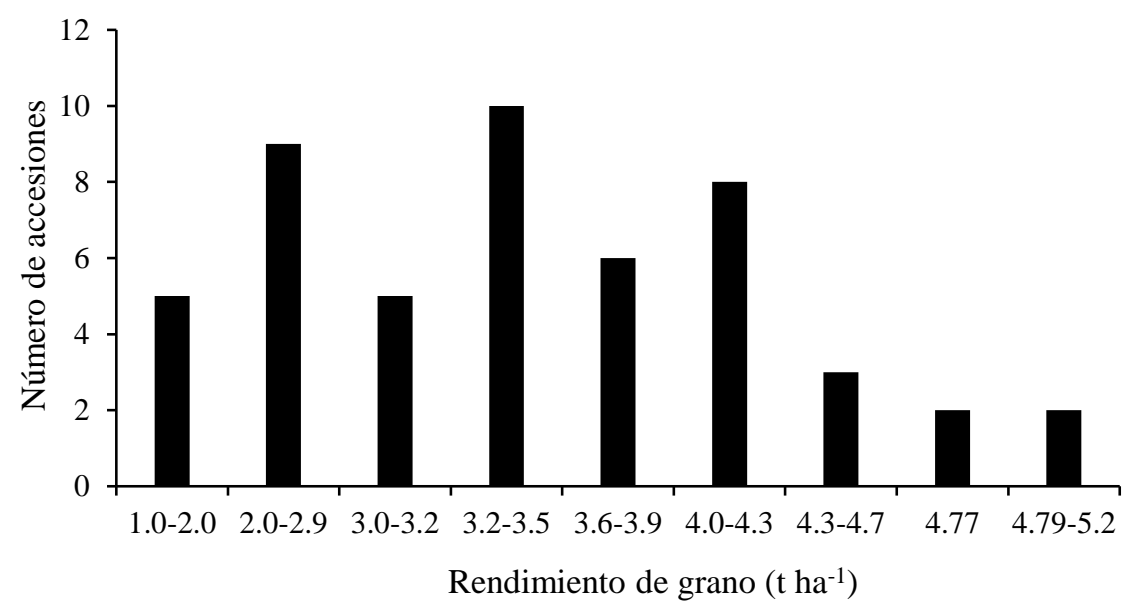

Figura 1. Distribución en frecuencia absoluta de las accesiones evaluadas por su rendimiento de grano. 
Estos valores superan la expectativa que se tenía de la raza, considerando que los maíces palomeros de Brasil tienen rendimientos menores a $2.5 \mathrm{t} \mathrm{ha}^{-1}$ (Vieira et al., 2016); sin embargo, aún no son capaces de competir en el mercado nacional, pues la variedad comercial de maíz palomeroV-460P, rinde $5 \mathrm{t} \mathrm{ha}^{-1}$ en riego y más de $2.3 \mathrm{t} \mathrm{ha}^{-1}$ en temporal en la zona de las Huastecas (Valadez-Gutiérrez et al., 2014).

El rendimiento de grano promedio que se obtuvo en esta investigación fue ligeramente menor a los valores que presentaron otros materiales de maíces nativos cultivados y evaluados en condiciones de temporal en el Valle de México (González et al., 2007), por lo que es posible que en temporal Palomero Toluqueño tenga rendimientos inferiores a otros materiales y sea una de las causas por la cual se deje de cultivar.

\section{Características del reventado}

El volumen de expansión del grano osciló entre 1 y $7.25 \mathrm{~cm}^{3} \mathrm{~g}^{-1}$ con una media de $2.73 \mathrm{~cm}^{3} \mathrm{~g}^{-1}$ (Cuadro 4); sin embargo, ninguno de los materiales evaluados fue cercano al volumen de expansión del testigo (Figura 2) y menos aún a los maíces palomeros mejorados de los Estados Unidos de América, con volúmenes de expansión de 35 a $55 \mathrm{~cm}^{3} \mathrm{~g}^{-1}$ (Dofing et al., 1990; Sakin et al., 2005) o los materiales brasileños con medias de $18.7 \mathrm{~cm}^{3} \mathrm{~g}^{-1}$ (Miranda et al., 2003); incluso, estuvieron por debajo de $11.4 \mathrm{~cm}^{3} \mathrm{~g}^{-1}$ que es la media del volumen de reventado de la raza Chapalote (Vázquez et al., 2011).

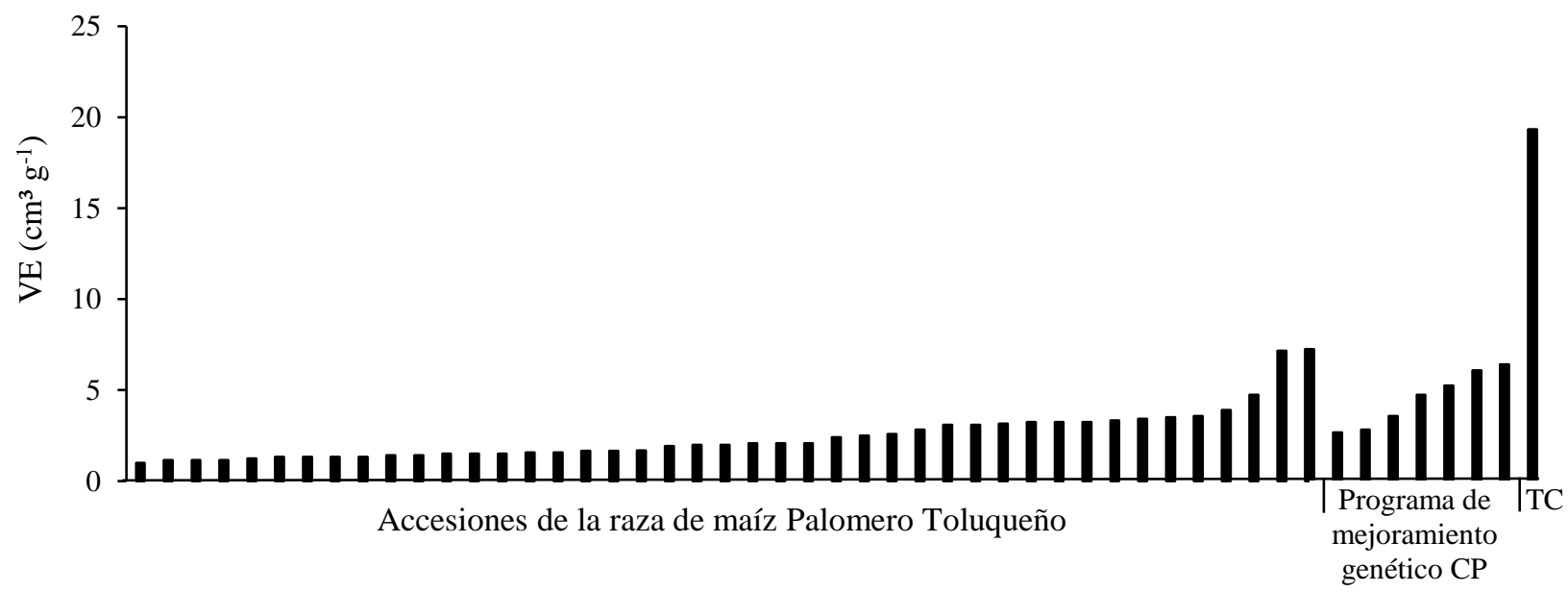

Figura 2. Distribución en frecuencia absoluta de las accesiones evaluadas y del testigo comercial (TC) por volumen de expansión.

La diferencia entre el volumen de expansión de los materiales resguardados en los bancos de germoplasma del Instituto Nacional de Investigaciones Forestales, Agrícolas y Pecuarias (INIFAP) y los maíces de los custodios no fue significativa, pese a no tener el mismo origen; esto puede ser indicativo de que la capacidad de expansión de la raza no se ha modificado en forma significativa a través del tiempo. Lo anterior, puede ser atribuible a la selección realizada por los productores en su troje, donde buscan mazorcas parecidas a Palomero Toluqueño, aunque en la siembra sea necesario asociarlos con maíces de grano harinoso que mejoren la calidad de uso para tortilla (Gámez et al., 2014). 
La media del volumen de expansión por hectárea $\left(8.86 \mathrm{~m}^{3} \mathrm{ha}^{-1}\right)$ es similar a la obtenida en materiales de otros países (Berilli et al., 2013; Vieira et al., 2016), aunque dentro de los materiales evaluados se encontraron algunos sobresalientes para esta variable, tales como: Pinto de Amarillo, Criollo Plaza, Palomero Esbelto entre otros (Cuadro 4).

Cuadro 4. Comparación de medias de cinco variables de las mejores 10 y peores cinco accesiones en su volumen de expansión por hectárea y el testigo comercial.

\begin{tabular}{cccccc}
\hline Accesión & REND $\left(\mathrm{t} \mathrm{ha}^{-1}\right)$ & VE $\left(\mathrm{cm}^{3} \mathrm{~g}^{-1}\right)$ & PP $(1-5)$ & PGSR $(\%)$ & VEH $\left(\mathrm{m}^{3} \mathrm{ha}^{-1}\right)$ \\
\hline NayPP-II × Mex-5-77 & $4.15 \mathrm{a}$ & $6.08 \mathrm{~b}$ & $1.88 \mathrm{j}$ & $58.64 \mathrm{a}$ & $24.35 \mathrm{a}$ \\
Comp. Amarillo & $3.41 \mathrm{a}$ & $7.25 \mathrm{~b}$ & $1.75 \mathrm{k}$ & $53.8 \mathrm{~d}$ & $24.27 \mathrm{a}$ \\
Pal. Pinto Amarillo & $3.82 \mathrm{a}$ & $4.75 \mathrm{c}$ & $1.38 \mathrm{~m}$ & $59.29 \mathrm{a}$ & $18.43 \mathrm{a}$ \\
Criollo Plaza & $3.85 \mathrm{a}$ & $4.75 \mathrm{c}$ & $2.63 \mathrm{f}$ & $58.53 \mathrm{a}$ & $17.92 \mathrm{a}$ \\
Comp. Blanco & $3.23 \mathrm{a}$ & $5.25 \mathrm{~b}$ & $1.75 \mathrm{k}$ & $59.79 \mathrm{a}$ & $16.67 \mathrm{a}$ \\
Palomero Esbelto & $2.55 \mathrm{~d}$ & $6.42 \mathrm{~b}$ & $1.63 \mathrm{l}$ & $55.72 \mathrm{c}$ & $14.94 \mathrm{~b}$ \\
Palomero Tardío & $4.03 \mathrm{a}$ & $3.58 \mathrm{e}$ & $2.13 \mathrm{i}$ & $53.79 \mathrm{~d}$ & $14.34 \mathrm{~b}$ \\
Hgo-609 & $4.08 \mathrm{a}$ & $3.33 \mathrm{e}$ & $2.63 \mathrm{f}$ & $70.55 \mathrm{a}$ & $13.51 \mathrm{~b}$ \\
Criollo Encarnación & $3.79 \mathrm{a}$ & $3.25 \mathrm{e}$ & $2.88 \mathrm{~d}$ & $57.07 \mathrm{~b}$ & $12.87 \mathrm{~b}$ \\
Mex-5 & $3.12 \mathrm{a}$ & $3.58 \mathrm{e}$ & $2.75 \mathrm{e}$ & $59.76 \mathrm{a}$ & $11.94 \mathrm{~b}$ \\
Palomero Crema & $1.55 \mathrm{j}$ & $2.67 \mathrm{f}$ & $2.5 \mathrm{f}$ & $62.39 \mathrm{a}$ & $4.18 \mathrm{~h}$ \\
Pue-955 & $2.05 \mathrm{f}$ & $1.33 \mathrm{i}$ & $3.25 \mathrm{a}$ & $79.69 \mathrm{a}$ & $2.73 \mathrm{i}$ \\
ARR-1,2PL & $2.63 \mathrm{c}$ & 1.001 & $4.63 \mathrm{a}$ & $84.33 \mathrm{a}$ & $2.57 \mathrm{i}$ \\
ARR-81 & $2.02 \mathrm{f}$ & $1.17 \mathrm{l}$ & $4.88 \mathrm{a}$ & $81.81 \mathrm{a}$ & $2.35 \mathrm{i}$ \\
Pue-954 & $1.87 \mathrm{i}$ & $1.17 \mathrm{l}$ & $4 \mathrm{a}$ & $84.67 \mathrm{a}$ & $2.15 \mathrm{i}$ \\
Valle Verde & nd & $19.33 \mathrm{a}$ & $1 \mathrm{n}$ & $57.5 \mathrm{a}$ & $\mathrm{nd}$ \\
DSH 0.05 & 2.15 & 9.01 & 2.21 & 1.6 & 27.49 \\
\hline
\end{tabular}

Medias con letras iguales de columnas no son estadísticamente diferentes (Tukey, 0.05). DSH= diferencia significativa honesta. nd= no determinado.

Estas accesiones no son aptas en su estado actual para la comercialización, pues fue el rendimiento de grano y no el volumen de expansión la causante de la aparente superioridad, ya que la comercialización de los maíces palomeros se hace por volumen de maíz ya reventado (Soylu y Tekkanat, 2007).

Los valores de granos sin reventar fueron superiores al $50 \%$ y menores al $85 \%$, incluso en el palomero comercial. La accesión Pue-235 presentó menor porcentaje de grano sin reventar y ARR1,2PL-Blanco resultó sumamente baja en el porcentaje de granos sin reventar aun cuando su volumen de expansión fue el más bajo (Cuadro 4).

Los valores observados para esta variable son al menos cinco veces superiores a los reportados por Sweley et al. (2012) en híbridos comerciales de maíz palomero, haciendo que la raza Palomero Toluqueño en su estado actual, no pueda competir con los materiales existentes en el mercado, por lo que es necesario el establecimiento de un programa de mejoramiento genético para características de reventado. 
El hecho de que el grano de maíz palomero reviente no implica necesariamente buena calidad, ya que se espera que las rosetas sean de tamaño grande y que el pericarpio se pulverice casi en su totalidad (Ziegler, 2001). Al respecto, la pulverización del pericarpio en el testigo tuvo un valor de 1 , mientras que en los materiales nativos fue de 1.3 a 4.8 .

Dos accesiones de la raza Palomero Toluqueño presentaron valores inferiores a 1.8 indicativo de pequeños restos de pericarpio en las rosetas. En las nueve accesiones con valores de 4 para pulverización del pericarpio, la expansión se manifestó en la parte superior del grano, dejando más de $80 \%$ del pericarpio intacto. Los materiales de los custodios presentaron valores entre 2.3 y 3.1 (Cuadro 4), indicativo de que las semillas forman rosetas sin eliminar más de $40 \%$ del pericarpio.

La interacción genotipo $\times$ ambiente está ligada a la estabilidad de los genotipos a través de los ambientes (Cortes et al., 2000) en este sentido, las accesiones de Palomero Toluqueño cambiaron su respuesta en volumen de expansión por hectárea, volumen de expansión por muestra de 30 g y pulverización del pericarpio de acuerdo con el ambiente.

En estas variables los genotipos interactuaron variando en magnitud y dirección en las localidades (Cuadro 2). Mientras que rendimiento de grano, forma de la roseta y porcentaje de granos sin reventar no interactuaron significativamente con las localidades; por lo tanto, tienen mayor estabilidad a través de ambientes.

\section{Correlación entre las variables evaluadas}

El pericarpio pulverizado fue la variable con el mayor número de correlaciones significativas; mantuvo correlaciones negativas con el volumen de expansión por hectárea y por gramo de grano. Se observó además que el volumen de expansión tuvo una correlación negativa con el porcentaje de granos sin reventar (Cuadro 5), lo cual concuerda con el supuesto de que a mayor número de granos reventados y cantidad de pericarpio eliminado, mayor será el volumen de expansión (Hoseney et al., 1983); por lo tanto, el incremento en el volumen de expansión se asocia con una mejor palatabilidad de la roseta eliminando restos de pericarpio.

Cuadro 5. Coeficientes de correlación entre las características de reventado y rendimiento.

\begin{tabular}{cccccc}
\hline & PGSR & VEH & PP & FR & REND \\
\hline VE & $-0.585^{* *}$ & $0.7993^{* * *}$ & $-0.734^{* *}$ & -0.109 & -0.241 \\
PGSR & & $-0.685^{* *}$ & $0.808^{* *}$ & 0.177 & 0.193 \\
VEH & & $-0.769^{* *}$ & -0.122 & 0.281 \\
PP & & & 0.083 & 0.124 \\
FR & & & & -0.01 \\
\hline
\end{tabular}

${ }^{* *}=p \leq 0.01$.

En el aprovechamiento comercial de maíz palomero se ha observado una correlación negativa del rendimiento con el reventado (Dofing et al., 1991; Vieira et al., 2016), ambas características son de gran interés en los maíces palomeros (Ceylan y Karababa, 2001; Soylu y Tekkanat, 2007). En los materiales de Palomero Toluqueño evaluados el rendimiento no tuvo correlación significativa con ninguna variable relacionada con reventado, lo que dificulta la selección simultánea para 
ambos aspectos, por ello sería deseable involucrar índices de selección para mejorar de manera paralela características de diferente naturaleza, tal como lo han demostrado Berilli et al. (2013); Freitas et al. (2014); Vieira et al. (2016), los dos últimos específicamente en maíz palomero.

\section{Conclusiones}

Los materiales de la raza de maíz Palomero Toluqueño resguardados en los bancos de germoplasma y por agricultores custodios, en su estado actual, no cuentan con los atributos de reventado suficientes para competir con los palomeros comerciales en la elaboración de palomitas. Sin embargo, existen accesiones sobresalientes en rendimiento de grano y capacidad de expansión que permitirán iniciar programas de mejoramiento genético de la raza.

\section{Literatura citada}

Berilli, A. P. C. G.; Pereira, M. G.; Trindade, R. S.; Costa, F. R. and Cunha, K. S. 2013. Response to the selection in the $11^{\text {th }}$ cycle of reciprocal recurrent selection among full-sib families of maize. Acta Scientiarum Agronomy. 35(4):435-441. doi:10.4025/actasciagron.v35i4.17489.

Bidlack, J.; Malone, M. and Benson, R. 1992. Molecular structure and component integration of secondary cell walls in plants. Proceedings of the Oklahoma Academy of Science. 72:51-56.

Borras, F.; Seetharaman, K.; Yao, N.; Robutti, J. L.; Percibaldi, N. M. and Eyherabide. G. H. 2006. Relationship between popcorn composition and expansion volume and discrimination of corn types by using zein properties. Cereal Chemistry. 83(1):86-92. Doi: 10.1094/CC-830086.

Buenrostro-Robles, M.; Lobato-Ortiz, R.; García-Zavala, J. J. y Sánchez-Abarca, C. 2017. Rendimiento de líneas de maíz exótico irradiado con rayos gamma y de híbridos de cruza simple. Rev. Fitotec. Mex. 40(3):351-358.

Ceylan, M. and Karababa, E. 2001. Comparison of sensory properties of popcorn from various types and sizes of kernel. J. Sci. Food Agric. 82(1):127-133. doi:10.1002/jsfa.1000.

Cortes, J. M.; Ñustez, C. E.; Martínez, R. y Estrada, N. 2000. Análisis de la interacción genotipo por ambiente en papa (Solanum tuberosum ssp. andigena), a través de una metodología no paramétrica. Agron. Colomb. 17(1-3):43-48.

Dofing, S. M.; ĎCroz-Mason, N. and Thomas-Compton, M. A. 1991. Inheritance of expansion volume and yield in two popcorn $\times$ dent corn crosses. Crop Sci. 31(3):715-718. doi:10.2135/cropsci1991.0011183X003100030035x.

Dofing, S. M.; Thomas-Compton, M. A. and Buck, J. S. 1990. Genotype $\times$ popping method interaction for expansion volume in popcorn. Crop Sci. 30(1):62-65. doi:10.2135/cropsci1990.0011183X003000010014x.

Freitas, I. L. J.; Do-Amaral, J. R. A. T.; Freitas, J. R. S. P.; Cabral, P. D. S.; Ribeiro, R. M. and Gonçalves, L. S. A. 2014. Genetic gains in the UENF-14 popcorn population with recurrent selection. Genetics Mol. Res. 13(1):518-527. doi: 10.4238/2014.January.21.21.

Gámez, V. A. J.; de la O-Olan, M.; Santacruz, V. A. y López, S. H. 2014. Conservación in situ, manejo y aprovechamiento de maíz Palomero Toluqueño con productores custodios. Rev. Mex. Cienc. Agríc. 5(8):1519-1530. Doi: 10.29312/remexca.v5i8.832.

Gökmen, S. 2004. Effects of moisture content and popping method on popping characteristics of popcorn. J. Food Eng. 65(3):357-362. Doi: 10.1016/j.jfoodeng.2004.01.034. 
González, H. A.; Vázquez, G. L. M.; Sahagún, C. J.; Rodríguez, P. J. E. y Pérez, L. D. J. 2007. Rendimiento del maíz de temporal y su relación con la pudrición de mazorca. Agric. Téc. Méx. 33(1):33-42.

Hoseney, R. C.; Zeleznak, K. and Abdelrahman, A. 1983. Mechanism of popcorn popping. J. Cereal Sci. 1(1):43-52. Doi: 10.1016/S0733-5210(83)80007-1.

Kato-Y, T. A.; Mapes, S. C.; Mera, O. L. M.; Serratos, H. J. A. y Bye, B. R. A. 2009. Origen y Diversificación del Maíz. Una revisión analítica. Universidad Nacional Autónoma de México (UNAM)-Comisión Nacional para el Conocimiento y Uso de la Biodiversidad (CONABIO). México, DF. 115 p.

Lu, H. J.; Bernardo, R. and Ohm, H. W. 2003. Mapping QTL for popping expansion volume in popcorn with simple sequence repeat markers. Theor. Appl. Gen. 106(3):423-427. Doi: 10.1007/s00122-002-1041-y.

Ma, X. Q.; Tang, J. H.; Teng, W. T.; Yan, J. B.; Meng, Y. J. and Li, J. S. 2007. Epistatic interaction is an important genetic basis of grain yield and its components in maize. Mol. Breed. 20(1):41-51. Doi: 10.1007/s11032-006-9071-9.

Márquez-Sánchez, F. 2001. More on inbreeding on maize germplasm reproduction. Maydica. 46(3):167-169.

Matsuoka, Y.; Vigouroux, Y.; Goodman, M. M.; Sanchez, G. J.; Buckler, E. and Doebley, J. 2002. A single domestication for maize shown by multilocus microsatellite genotyping. Proceedings of the National Academy of Sciences of the United States of America. 99(9):6080-6084. doi: 10.1073/pnas.052125199.

Mauricio, S. R. A.; Figueroa, C. J. D.; Taba, S.; Reyes,V. M. L.; Rincón, S. F. y Mendoza, G. A. 2004. Caracterización de accesiones de maíz por calidad de grano y tortilla. Rev. Fitotec. Mex. 27(3):213-222.

Miranda, G. V.; Coimbra, R. R.; Souza, L. V.; Guimarães, M. L. J. and De Melo, V. A. 2003. Potencial de melhoramento e divergência genética de cultivares de milho-pipoca. Pesquisa Agropecuária Brasileira. 38(6):681-688. doi.org/10.1590/S0100-204X2003000600003.

Ortega, P. R. A.; Sánchez, G. J. J.; Castillo, G. F. y Hernández, C. J. M. 1991. Estado actual de los estudios sobre maíces nativos de México. In: R. Ortega P., G. Palomino H., F. Castillo G., V. A. González H. y M. Livera M. (eds.). Avances en el estudio de los recursos fitogenéticos de México. SOMEFI, AC. Chapingo, Estado de México. 161-185 pp.

Sakin, M. A.; Gokmen, S.; Yildirim, A.; Belen, S. and Kandemir. N. 2005. Effects of cultivar type on yield and quality of popcorn (Zea mays everta). New Zealand Journal of Crop and Horticultural Science. 33(1):17-23. doi.org/10.1080/01140671.2005.9514326.

Santacruz-Varela, A. 2001. Genetic diversity of North American popcorn and its relationship with Mexican and South American popcorns. Ph. D. Dissertation. Iowa State University. Ames, Iowa, USA. 326 p.

SAS. Institute. 2004. SAS/STAT ${ }^{\circledR} 9.1$ User's Guide. SAS Institute Inc. Cary, North Carolina, USA. $5121 \mathrm{p}$.

SMN. 2015. Servicio Meteorológico Nacional. Información climatológica por estado. Servicio Meteorológico Nacional. Comisión Nacional del Agua (CONAGUA). México, DF. http://smn.conagua.gob.mx./es/informacion-climatologica-por-estado.

Soylu, S. and Tekkanat, A. 2007. Interactions amongst kernel properties and expansion volume in various popcorn genotypes. Journal of Food Engineering. 80(1):336-341. Doi: 10.1016/j.jfoodeng.2006.06.00. 
Sweley, J. C.; Rose, D. J. and Jackson, D. S. 2012. Hybrid and environment effects on popcorn kernel physiochemical properties and their relationship to microwave popping performance. J. Cereal Sci. 55(2):188194. doi:10.1016/j.jcs.2011.11.006.

Valadez-Gutiérrez, J.; Gómez-Montiel, N. O.; Preciado-Ortiz, R. E.; Reyes-Méndez, C. A. y PeñaRamos, A. 2014. V460P, variedad de maíz palomero para la región de Las Huastecas. Rev. Mex. Cienc. Agríc. 5(7):1303-1308. Doi: 10.29312/remexca.v0i7.1112.

Vázquez, C. M. G.; Ortega, C. A.; Guerrero, H. M. J. y Coutiño, E. B. 2011. Evaluación bioquímica e industrial de razas nativas de maíz de la región serrana de Sonora. In: Preciado, O. R. E. y Montes, H. S. (Eds.). Amplitud, mejoramiento, usos y riesgos de la diversidad genética de maíz en México. Sociedad Mexicana de Fitogenética, AC. Chapingo, Estado de México. 97-142 pp.

Vieira, R. A.; Rocha, R.; Scapim, C. A.; Amaral, J. R. A. T. and Vivas, M. 2016. Selection index based on the relative importance of traits and possibilities in breeding popcorn. Gen. Mol. Res. 15(2):1-10. Gmr.15027719. Doi:10.4238/gmr.15027719.

Wellhausen, E. J.; Roberts, L. M.; Hernández, X. E. y Mangelsdorf, P. C. 1951. Razas de maíz en México. Su origen características y distribución. Secretaría de Agricultura y Ganadería. México, D. F. Folleto técnico núm. 5.237 p.

Ziegler, K. E. 2001. Popcorn. In: Hallauer, A. R. (Ed.). Specialty corns. CRC Press. Boca Raton, FL. 199-234 pp. 